\title{
FIBRAS SOLÚVEIS NO TRATAMENTO DA DOENÇA HEPÁTICA GORDUROSA NÃO-ALCOÓLICA: estudo piloto
}

\author{
Raquel ROCHA ${ }^{1}$, Helma P. COTRIM² ${ }^{2}$, Ana Cristina SIQUEIRA² e Shirley FLORIANO²
}

\begin{abstract}
RESUMO - O presente estudo piloto avaliou a eficácia de fibras solúveis no tratamento da doença hepática gordurosa não-alcoólica. Foram incluídos 12 pacientes que receberam $10 \mathrm{~g} /$ dia de fibras solúveis oral por 3 meses. Após o tratamento, observou-se redução nos valores do índice de massa corporal, circunferência da cintura e resistência à insulina em $100 \%$ dos casos, redução nos níveis de colesterol em $66,7 \%$ e normalização de ALT, AST e GGT em 75\%. Os resultados sugerem que a utilização de fibras solúveis pode colaborar no controle de fatores de risco e das enzimas hepáticas em pacientes com doença hepática gordurosa não-alcoólica e estimulam a realização de estudos controlados envolvendo controle histológico.
\end{abstract}

DESCRITORES - Fígado gorduroso. Fibra alimentar.

\section{INTRODUÇÃO}

A doença hepática gordurosa não-alcoólica (DHGNA) é uma das mais freqüentes doenças do fígado da atualidade. Está relacionada a diversos fatores de risco e um amplo espectro clínico-histológico, que inclui a esteatose, a esteatohepatite com e sem fibrose e cirrose.

A DHGNA é comumente observada em pacientes obesos, diabéticos e com hiperlipidemia, entretanto estão também entre seus fatores de risco medicamentos, cirurgias abdominais, toxinas ambientais e doenças genéticas ${ }^{(4)}$.

$\mathrm{O}$ tratamento de DHGNA envolve principalmente o controle dos fatores de risco. Assim, a recomendação de dietas equilibradas e atividade física regular são consideradas essenciais, principalmente quando a doença se relaciona com obesidade, dislipidemia e diabetes mellitus. Entretanto, como estas medidas nem sempre são eficazes em longo prazo, a introdução de drogas como parte do tratamento tem sido avaliada. Entre elas estão o ácido urso-desoxicólico, os antioxidantes (alfa-tocoferol, vitaminas B e C) e as drogas insulinosensibilizantes ${ }^{(1)}$. Embora haja resultados promissores, os ensaios terapêuticos com medicamentos são ainda insuficientes para resposta conclusiva sobre sua eficácia e, por isso, consideram-se de interesse estudos que propõem a utilização de novas drogas.

Como a eficácia das fibras solúveis tem sido observada no controle de condições metabólicas, notadamente da obesidade e dislipidemia, que são fatores de risco relevantes para a DHGNA, este estudo teve como objetivo principal determinar a eficácia da suplementação oral de fibras no tratamento dessa doença hepática.

\section{MÉTODOS}

Ensaio clínico piloto, desenvolvido no Ambulatório de Esteatohepatite Não-Alcoólica (NASH) do Hospital Universitário Professor Edgard Santos, Universidade Federal da Bahia (UFBA), Salvador, BA.

\section{Critérios de inclusão}

Pacientes com diagnóstico de DHGNA, segundo os seguintes parâmetros: história de ingestão ocasional ou negativa de etanol $(<140 \mathrm{~g}$ de etanol/semana); exclusão de outras doenças crônicas do fígado (vírus $\mathrm{B}$ e $\mathrm{C}$ da hepatite, hemocromatose e autoimunes); métodos de imagem (ultra-sonografia ou tomografia computadorizada ou ressonância magnética), mostrando esteatose hepática; elevação dos níveis das aminotransferases (ALT/AST e GGT).

\section{Critérios de exclusão}

Idade inferior a 18 anos; mulheres grávidas; diabéticos em uso de insulina ou hipoglicemiantes orais; pacientes em tratamento medicamentoso para doenças crônicas sistêmicas ou infecciosas; pacientes que haviam realizado algum tratamento medicamentoso para perda de peso e diabetes nos últimos 6 meses; pacientes com história de

${ }^{1}$ Escola de Nutrição e ${ }^{2}$ Faculdade de Medicina, Universidade Federal da Bahia, Salvador, BA.

Correspondência: Dra. Raquel Rocha - Avenida Araújo Pinho, 32 - Canela - 40110-150 - Salvador, BA. E-mail: raquelrocha2@yahoo.com.br 
intolerância a fibras; assinatura de consentimento livre e esclarecido para participar do estudo. O projeto foi aprovado pelo Comitê de Ética em Pesquisas da Maternidade Climério de Oliveira e Programa de Pós-Graduação em Medicina e Saúde, UFBA.

A fibra solúvel utilizada é derivada de cutículas do plantago ovata (Ispaghula husk), flavorizado (laranja), sem adição de açúcar, na dose de $10 \mathrm{~g}$ diárias (Plantaben ${ }^{\circledR}$ - Altana Pharma), distribuídas em duas doses de $5 \mathrm{~g}, 30$ minutos antes das principais refeições, por 3 meses. A droga é bem tolerada e não está associada com eventos adversos sérios, sendo descritas flatulência e sensação de plenitude, que desaparecem com a suspensão da droga. As contra-indicações são hipersensibilidade ao plantago ovata e diagnóstico de obstrução intestinal.

\section{Seguimento}

Os pacientes foram acompanhados mensalmente através de consultas clínicas e realização de exames laboratoriais: aspartato aminotransferase (AST), alanina aminotransferase (ALT), gama - glutamiltransferase (GGT); fosfatase alcalina (FA), triglicerídeos (TG), colesterol total, glicemia de jejum e insulina em jejum. A resistência à insulina foi avaliada pelo modelo matemático Homeostasis Model Assessment Method (HOMA-IR). Peso e altura foram avaliados para identificação do índice de massa corporal (IMC) e foi aferida a medida da circunferência da cintura (CC). Para avaliação desses parâmetros (IMC e CC) foram utilizados os valores de referência da OMS (1997). Os dados foram analisados pelo pacote estatístico SPSS, com utilização Wilcoxon para comparar as variáveis, considerando os níveis de significância estatística de 5\% de possibilidade de erro tipo alfa.

\section{RESULTADOS}

Vinte pacientes foram selecionados para o estudo, 13 preencheram os critérios exigidos pelo protocolo e 12 concluíram o estudo. Um paciente foi excluído porque apresentou diarréia como efeito colateral da droga. A idade média foi de 40,3 $\pm 8,7$ anos e $92,2 \%$ eram do sexo masculino. Na avaliação antropométrica, todos pacientes apresentavam excesso de peso (IMC $\geq 25 \mathrm{~kg} / \mathrm{m}^{2}$ ) e 11 apresentavam obesidade central. Observou-se redução nos valores médios do IMC e circunferência da cintura dos 12 pacientes que concluíram o tratamento, mas sem significância estatística (Tabela 1). A glicemia sérica e o valor de HOMA também tiveram redução, entretanto sem significância estatística (Tabela 1). No lipidograma, encontraram-se seis pacientes com hipercolesterolemia e sete com hipertrigliceridemia; houve aumento nos níveis séricos de colesterol e triglicérides, $P>0,05$ (Tabela 1). Isoladamente, observou-se redução nos níveis de colesterol sérico em 33,3\% (4/12) dos pacientes e de triglicérides em 50,0\% (6/12) dos casos. A normalização dos níveis séricos de ALT, AST e GGT foram observadas em
$75 \%$ (9/12) dos casos. A diferença entre os níveis séricos destas enzimas hepáticas no inicio e término do tratamento foi estatisticamente significante (Tabela 1).

TABELA 1. Comparação dos dados antropométricos e laboratoriais, no início e ao final do tratamento com fibras solúveis, em pacientes com doença hepática gordurosa não-alcoólica

\begin{tabular}{lccc}
\hline Variáveis & $\begin{array}{c}\text { Antes do tratamento } \\
\text { com fibras solúveis } \\
\text { Média } \pm \text { DP }\end{array}$ & $\begin{array}{c}\text { Ao final do tratamento } \\
\text { com fibras solúveis } \\
\text { Média } \pm \text { DP }\end{array}$ & $\boldsymbol{P}$ \\
\hline IMC $\left(\mathrm{kg} / \mathrm{m}^{2}\right)$ & $30,7 \pm 3,5$ & $29,7 \pm 2,9$ & 0,1 \\
CC $(\mathrm{cm})$ & $99,2 \pm 7,9$ & $97,6 \pm 6,4$ & 0,9 \\
AST $(\mathrm{U} / \mathrm{L})$ & $48,1 \pm 19,7$ & $30,8 \pm 13,3$ & 0,01 \\
ALT $(\mathrm{U} / \mathrm{L})$ & $99,2 \pm 41,1$ & $55,9 \pm 30,7$ & 0,02 \\
GGT $(\mathrm{U} / \mathrm{I})$ & $72,2 \pm 37,4$ & $43,3 \pm 21,7$ & 0,05 \\
Glicemia $(\mathrm{mg} / \mathrm{dL})$ & $98,8 \pm 11,5$ & $94,5 \pm 14,6$ & 0,4 \\
HOMA & $3,2 \pm 1,1$ & $2,4 \pm 1,2$ & 0,1 \\
Colesterol $(\mathrm{mg} / \mathrm{dL})$ & $195,6 \pm 53,8$ & $196,6 \pm 35,8$ & 0,6 \\
Triglicérides $(\mathrm{mg} / \mathrm{dl})$ & $167,6 \pm 59,0$ & $186,0 \pm 67,4$ & 0,3 \\
\hline
\end{tabular}

DP - desvio-padrão; IMC - índice de massa corporal;
CC - circunferência da cintura; HOMA - "homeostasis model assessment method"

\section{DISCUSSÃO}

A redução nos valores do $\mathrm{IMC}$, circunferência da cintura e resistência à insulina em $100 \%$ dos casos, redução nos níveis de colesterol sugerem controle dos fatores metabólicos relacionados à DHGNA após o tratamento com as fibras solúveis. A normalização nos níveis séricos das enzimas hepáticas, na maioria dos pacientes (75\%) é também resultado relevante, pois sugere controle da atividade da doença DHGNA.

O mecanismo de ação das fibras solúveis não foi completamente elucidado. Entretanto, tem sido atribuída às fibras, interferência no metabolismo dos lipídeos, que impedem a re-esterificação de ácidos graxos no tecido hepático e induzem a uma menor síntese e secreção de triacilglicerois ${ }^{(2)}$. Alguns trabalhos em ratos têm mostrado efeito protetor das fibras solúveis no desenvolvimento da esteatose hepática sem qualquer efeito sobre a hipertrigliceridemia ${ }^{(2,3,5)}$. DAUBIOUL et al. ${ }^{(3)}$ estudando o efeito da suplementação de fibra solúvel em ratos obesos Zucker, observaram neutralização na formação de tecido adiposo e esteatose hepática, sem alterar triglicérides e glicose séricos e sugeriram o chamado efeito "hepatoprotetor" das fibras solúveis.

Na presente investigação duas metas desejadas no tratamento da DHGNA foram alcançadas: controle dos fatores de risco e da resistência à insulina, e normalização dos níveis das enzimas hepáticas (AST, ALT, GGT), sugerindo redução à agressão hepatocelular.

Estes resultados estimulam a realização de estudos controlados com esta droga, envolvendo também a avaliação histológica da DHGNA, doença hepática de elevada freqüência e para a qual não estão ainda disponíveis esquemas terapêuticos definidos. 
Rocha R, Cotrim HP, Siqueira AC, Floriano S. Non alcoholic fatty liver disease: treatment with soluble fibres. Arq Gastroenterol. 2007;44(4):350-2.

ABSTRACT - The pilot study evaluated the efficiency of oral soluble fibers to treat patients with nonalcoholic fatty liver disease. Twelve patients received $10 \mathrm{~g} /$ day of soluble fibers during 3 months. After the treatment $100 \%$ of patients presented reduction in body mass index, waist circumference and insulin resistance index. In $66.7 \%$ of the patients were observed reduction of the cholesterol levels and $75 \%$ presented normal liver enzymes (AST, ALT, and GGT). The present study suggests that oral soluble fibers may be useful to control risk factors and liver enzymes in patients with nonalcoholic fatty liver disease. However, future studies with histological controls are considered necessary.

HEADINGS - Fatty liver. Dietary fiber.

\section{REFERÊNCIAS}

1. Angulo P, Lindor KD. Treatment of nonalcoholic fatty liver: present and emerging therapies. Sem Liver Dis. 2001;21:81-8.

2. Beylot M. Effects of inulin-type fructans on lipid metabolism in man and in animal models. Br J Nutr. 2005;93(Suppl 1):s163-s8.

3. Daubioul CA, Taper HS, De Wispelaere LD, Delzenne NM. Dietary oligofructose lessens hepatic steatosis, but does not prevent hypertriglyceridemia in obese Zucker rats. J Nutr. 2000;130:1314-9.
4. Day CP, James OEW. Hepatic steatosis: innocent bystander or guilty party? Hepatology. 1998;27:1463-6.

5. Delzenne NM, Daubioul C, Neyrink A, Lasa M, Taper HS. Inulin and oligofructose modulate lipid metabolism in animals: review of biochemical events and future prospects. Br J Nutr. 2002;87(Suppl 2):s255-s9. 